\title{
Adhesive penetration of hardwood A generic penetration model
}

\section{Journal Article}

\section{Author(s):}

Mendoza, Miller; Hass, Philipp; Wittel, Falk K.; Niemz, Peter; Herrmann, Hans J.

Publication date:

2012-01

Permanent link:

https://doi.org/10.3929/ethz-b-000045867

\section{Rights / license:}

In Copyright - Non-Commercial Use Permitted

\section{Originally published in:}

Wood Science and Technology 46(1-3), https://doi.org/10.1007/s00226-011-0422-2 


\title{
Adhesive penetration of hardwood: a generic penetration model
}

\author{
Miller Mendoza • Philipp Hass • Falk K. Wittel • \\ Peter Niemz • Hans J. Herrmann
}

Received: 17 March 2010/Published online: 15 May 2011

(C) Springer-Verlag 2011

\begin{abstract}
An analytical model to predict the penetration of adhesives into hardwood is proposed. Penetration into hardwood is dominated by the vessel network which prohibits porous medium approximations. The model considers two scales: (1) a one dimensional capillary fluid transport of a hardening adhesive through a single, straight vessel with diffusion of solvent through the walls of the vessel; and (2) a mesoscopic scale based on topological characteristics of the vessel network. Given an initial amount of adhesive and applied bonding pressure, the portion of the filled structure could be calculated. The model was applied to beech samples joined with three different types of adhesive (PUR, UF, PVAc) under various growth ring angles as described by Hass et al. (2011). The model contains one free parameter that can be adjusted in order to fit the experimental data.
\end{abstract}

\section{List of symbols}

\section{Adhesive parameters}

$\eta_{g} \quad$ Viscosity of the fluid without any hardening process (mPa s)

$\gamma \quad$ Viscosity parameter fit with experiments (no units)

$\alpha \quad$ Viscosity parameter fit with experiments $\left(\mathrm{s}^{-1}\right)$

$\beta \quad$ Viscosity parameter fit with experiments $\left([\mathrm{C}]^{-1}\right.$ )

$\eta \quad$ Viscosity of the adhesive (mPa s)

$a_{1}$ Viscosity parameter fit with experiments $\left(\mathrm{s}^{-1}\right)$

$b_{2} \quad$ Viscosity parameter fit with experiments (no units)

$c_{1} \quad$ Viscosity parameter fit with experiments (s)

$d_{1}$ Viscosity parameter fit with experiments $\left(\mathrm{mPa} \mathrm{s}^{2}\right)$

$\sigma \quad$ Surface tension $(\mathrm{N} / \mathrm{m})$

M. Mendoza $(\bowtie) \cdot$ P. Hass · F. K. Wittel · P. Niemz · H. J. Herrmann Institute for Building Materials, ETH Zurich, Schafmattsr. 6, 8093 Zurich, Switzerland e-mail: mmendoza@ethz.ch 
$\theta_{c} \quad$ Contact angle between adhesive and cell walls $\left({ }^{\circ}\right)$

\section{Wood structure parameters}

$R \quad$ Radius of the vessels (m)

$K \quad$ Wave number for the single wavy vessels $\left(\mathrm{m}^{-1}\right)$

$N=a / 4 \quad$ Amplitude of the wavy vessel (m)

$\lambda \quad$ Wavelength for the single wavy vessels (m)

A Vessel cross section $\left(\mathrm{m}^{2}\right)$

$V_{u} \quad$ Volume of a single unit cell $\left(\mathrm{m}^{3}\right)$

$x_{T, R, L} \quad$ Tangential, radial, and longitudinal coordinates $(\mathrm{m})$

$s_{b} \quad$ Path along one radial wave (m)

$s_{\text {total }} \quad$ Total path along the network radial wave $(\mathrm{m})$

$L_{T, R, L} \quad$ Tangential, radial, and longitudinal lengths of the sample (m)

$N_{\text {uL,uR,uT }} \quad$ Number of unit cells along the longitudinal, radial, and tangential directions (no units)

$N_{\text {uLf,uRf,uTf }}$ Number of unit cells on the RT, LT, and RL planes (no units)

$N_{v} \quad$ Number of vessels in the plane $R-T$ (no units)

$a \quad$ Maximum distance between two consecutive vessels (m)

$b \quad$ Wavelength of the vessels (m)

$c \quad$ Maximum distance between two consecutive radial waviness (m)

$d \quad$ Wavelength of the radial waviness $(\mathrm{m})$

$g_{T, R} \quad 1+\left(a^{2} \pi^{2} / 16 b^{2}\right), 1+\left(c^{2} \pi^{2} / 8 d^{2}\right)$ (no units)

$\theta \quad$ Deviation angle of the vessels respect to the long. axis $\left({ }^{\circ}\right)$

$\psi \quad$ Growth ring angle $\left(^{\circ}\right)$

\section{Process parameters}

$t \quad$ Time (s)

$x, y, z \quad$ Local coordinate system for a single vessel description (m)

$R, \theta, z \quad$ Cylindrical coordinate system for a single vessel description $(\mathrm{m}, \stackrel{\circ}{ }, \mathrm{m})$

$X, Y, Z \quad$ Global coordinate system (m)

$L, R, T \quad$ Body fixed coordinate system (m)

$l \quad$ Penetrated distance inside a single cylindrical vessel $(\mathrm{m})$

$l_{v} \quad$ Height of the single wavy vessels (m)

$\delta_{L, R, T} \quad$ Maximum penetration depths in the longitudinal, radial, and tangential directions (m)

$\delta_{\mathrm{Lh}, \mathrm{Rh}, \mathrm{Th}}$ Maximum penetration depths in the longitudinal, radial, and tangential directions with hardening process $(\mathrm{m})$

$\delta_{V} \quad$ Maximum penetration depth for volume limiting factor (m)

$\delta_{h} \quad$ Maximum penetration depth for hardening process limiting factor $(\mathrm{m})$

$\underline{\delta} \quad$ Penetration vector in the system $(R-T-L)(\mathrm{m})$

$\Delta_{L, R, T} \quad$ Tangential, longitudinal, and radial components of the penetration vector (m)

$V=V_{p} \quad$ Volume of adhesive inside a single vessel $\left(\mathrm{m}^{3}\right)$

$V_{T} \quad$ Total volume of adhesive inside the network $\left(\mathrm{m}^{3}\right)$

$C, C_{0} \quad$ Concentration, initial concentration of the solvent [C]

$N_{0} \quad$ Initial amount of solvent $\left(\mathrm{m}^{3}\right)$ 
$D \quad$ Diffusivity of the solvent through cell walls $\left(\mathrm{m}^{2} / \mathrm{s}\right)$

$P_{A} \quad$ Applied pressure to the adhesive $(\mathrm{Pa})$

$\underline{\underline{M}} \quad$ Rotation matrix (no units)

\section{Introduction}

The most important principle in timber engineering when producing structural wood components of constant quality consists of cutting wood into smaller pieces, selecting the best ones, and joining them again by adhesive bonds. What is known as a rather simple processing step becomes quite complicated, once it is studied in detail as the penetration of the hardening adhesive into the porous wood skeleton. The details of the adhesive penetration can influence bond performance in multiple ways what determines the overall performance of structural parts (Marra 1992; Custodio et al. 2009). Among other parameters, the interplay between pore space geometry and fluid transport, cell wall material and adhesive rheology and, of course, process parameters like amount of adhesive, growth ring orientation, and surface roughness, strongly influence studies on adhesive penetration (Kamke and Lee 2007). While for softwood predictions are rather simple, the microstructure of hardwoods complicates the problem significantly, since adhesives can penetrate through the big vessel network deeply into the wood structure (Siau 1984). While the topological characteristics of the vessel network in beech has been quantified by Hass et al. (2010), the authors showed in Hass et al. (2011) how the problem is dominated by the flow through the vessel network.

In the past, adhesive penetration into wood was studied experimentally or in descriptive form, e.g., by Wang and Yan (2005), Kamke and Lee (2007), Sernek et al. (1999), Niemz et al. (2004) and Collett (1972). For softwood, the penetration depth can be expressed by a simple trigonometric function describing the filling of cut tracheids (Suchsland 1958).

In this manuscript, an analytical model, based on typical network properties is derived to predict adhesive penetration and saturation of the vessel pore space. The model has two scales: the first scale describes the transport of a hardening adhesive through a single vessel in time due to an applied pressure and capillary effects. It also includes the possibility of a constant diffusion of solvent (water) through the vessel wall, which is an important process for some adhesives like PVAc or UF resins. When the viscosity increases by hardening and/or loss of solvent (water), the adhesive front slows down and finally stops. On the second (network) scale, the results for single vessels are embedded into a network model with characteristic topological properties like pore size distribution and connectivity taken from Hass et al. (2011). First, the rheological models of the adhesives are described, followed by the calculation of the penetration into a single vessel with solvent diffusion into the cellular structure through vessel walls. Subsequently, the network construction and the considered process parameters are discussed. With all these model components available, the model is compared to experiments where specimens are 
bonded with parallel longitudinal axes under variation of the growth ring angles using three different adhesive systems (PUR, UF, and PVAc).

\section{Description of the model}

Adhesive penetration is the result of the interplay of adhesive hardening, capillary penetration, and technological processing. In order to set up a model for adhesive penetration into hardwood, several models have to be combined in a hierarchical way. First, the evolution of the bulk viscosity of adhesives due to generic hardening mechanisms is addressed. On the fundamental level, the penetration of a fluid into one single, straight or wavy pipe is modeled. The model is enriched by diffusive transport of the solvent through the wall of the vessels. On the next hierarchic level, the fundamental model is applied to a network structure of perfectly aligned hardwood that represents the vessel network. Finally, the result of the vessel network penetration is rotated in order to consider the general situation, where the adhesive surface is not necessarily aligned to the material orientation. In a parametric study, it is also shown that material parameters like porosity, hardening time or applied amount of adhesive limit penetration.

\section{Modeling the hardening process}

The solidification process of various adhesives can be described by the increase of the viscosity $\eta$ with time $t$. Depending on the type of reaction during solidification, different viscosity models need to be applied. For example, reactive adhesives do not depend on the solvent concentration, while the viscosity increase of solventbased adhesives strongly depends on solvent concentration $C$. Hass et al. (2011) reported on experimental viscosity measurements for UF, PVAc, and PUR. If the solvent concentration is important, like in the case of PVAc, the viscosity evolution can be expressed by

$$
\eta(C, t)=\eta_{g}(C)[1+\gamma(C) \exp (\alpha(C) t)] \exp (\beta[1-C]),
$$

where $\gamma, \alpha$, and $\beta$ are parameters that depend on the adhesive type and the initial solvent concentration, and $\eta_{g}$ denotes the viscosity of the fluid without hardening. For PVAc $\gamma=\alpha=0$, since the solidification process is caused by the loss of moisture, and since the initial viscosity only depends on the initial concentration. For PUR adhesives, the same expression can be used, however, the concentration is kept constant during the process, expressed by $\beta=0$. In this case, $\eta_{g}, \gamma$, and $\alpha$ only depend on the initial concentration.

Unfortunately, hardening adhesives cannot be described by Eq. 1, since their hardening process is more complex. For example, the UF adhesive changes from liquid phase to gel phase during penetration, resulting in penetration arrest. The only active processes after this phase transition are the chemical curing reactions. Therefore, the viscosity model should take into account the critical time when the phase transition occurs. Additionally, the concentration of the solvent (water) 
changes with time due to the diffusion of the solvent into the cell wood structure. In order to describe these effects, the following viscosity relation is proposed:

$$
\eta_{U F}(C, t)=d_{1} \frac{\exp \left(b_{2}\left(1-\exp \left(-\frac{a_{1}}{t}\right)\right)\right)}{c_{1}-t},
$$

where $a_{1}, b_{2}, c_{1}$, and $d_{1}$ are experimental parameters. Using the data from Hass et al. (2011) $d_{1}=7.0 \times 10^{4} \mathrm{mPa} \mathrm{s}{ }^{2}$ and $a_{1}=1.05 \times 10^{4} \mathrm{~s}$ were found, while the two variable parameters $b_{2}$ and $c_{1}$ depend on the initial solvent concentration. $c_{1}$ describes the time when the penetration process finishes due to the liquid-gel transition. Using these two generic hardening models, the viscosity evolution of numerous adhesives can be described.

Penetration into single vessels

The fundamental scale is given by the capillary transport of a fluid characterized by its viscosity $\eta$, inside a straight single vessel of radius $R$ (Washburn 1921) with a penetration rate $\mathrm{d} l / \mathrm{d} t$ that follows

$$
\frac{\mathrm{d} l}{\mathrm{~d} t}=\frac{\mu}{8 \eta l} R^{2},
$$

where $\mu=P_{A}+2 \sigma \cos \left(\theta_{c}\right) / R$ with the applied pressure $P_{A}$, the surface tension $\sigma$ and the contact angle between fluid and pipe wall $\theta_{c}$. The penetration distance $l(t)$ is obtained by integration

$$
l(t)=\frac{R}{2} \sqrt{\mu \int_{0}^{t} \frac{1}{\eta(t)} \mathrm{d} t},
$$

leading to a total fluid volume of $V_{p}=\pi R^{2} l(t)$ inside the pipe. For reactive adhesives, whose hardening behavior only depends on time, the integral can be found by combining Eqs. 1 and 4 to

$$
l(t)=\frac{R}{2} \sqrt{\mu}\left(\frac{\alpha t-\log (1+\gamma \exp (\alpha t))}{\alpha \eta_{g} \exp (\beta(1-C))}\right)^{\frac{1}{2}} .
$$

In this way, the time-dependent penetration distance in one single straight vessel is obtained taking into account the applied pressure, the capillarity effects, and the reactive hardening process. In fact, due to surface roughness, mechanical and hygric responses of the cellular structure, the applied pressure does not necessarily have to be equal to the pressure on the adhesive. However, for simplicity this is not taken into account in this model. Additionally, for adhesives, whose viscosity changes during contact with wood, Eq. 4 cannot be integrated so easily, since the viscosity also depends on the concentration that changes with time. Changes of the contact angle and the surface tension of the adhesives with different solvent concentration are not considered in this work.

Since hardwood vessels are not straight, but weave tangentially around the rays, the penetration distance in $L$-direction needs to be modified. One possibility is to 


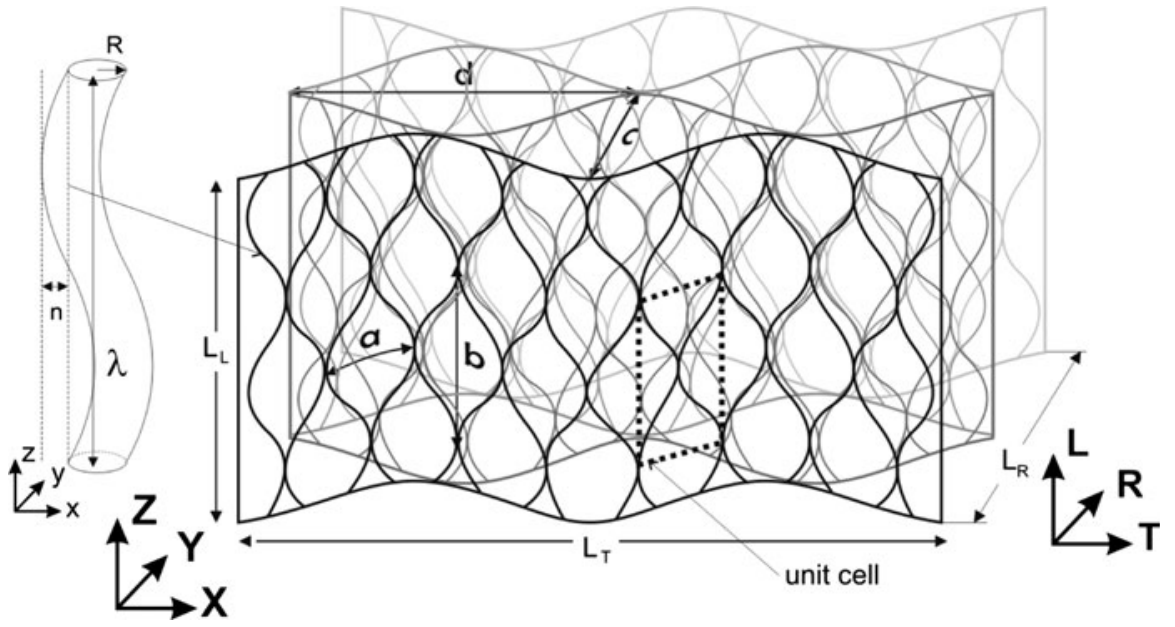

Fig. 1 left Single vessel geometry with radius $R$, amplitude $n=a / 4$ and vessel wavelength $\lambda=b$. right Vessel network of size $L_{T, R, L}$, and the unit cell that can reproduce the whole network. The network geometry is described by the parameter $a$ for the distance between two consecutive vessels, the vessel wavelength $b=\lambda$ and radial wavelength $d$, as well as the distance between consecutive layers $c$. Vessels are described in $x, y, z$ coordinates, the network in $T, R, L$ coordinates, while the global coordinate system is $X, Y, Z$

calculate the volume of a wavy vessel and compare it to the volume occupied by the adhesive in the straight one. To calculate the volume of the vessels by the radius $R$, wavelength $\lambda$, and amplitude $n$ (see Fig. 1) of the oscillation in the $z-y$-plane, the following parameterized form is proposed to describe the cross section of the vessel in the $x-y$-plane:

$$
x^{2}+\frac{[y-n \cos (k z)]^{2}}{\sec ^{2}[\arctan (n k \sin (k z))]}=R^{2},
$$

where the wave number is $k=2 \pi / \lambda$. By integrating the vessel area along the $z$ direction, the volume is obtained as

$$
\begin{aligned}
V\left(l_{v}\right) & =\int_{0}^{l_{v}} \pi R^{2} \sec [\arctan (n k \sin (k z))] \mathrm{d} z \\
& \simeq \pi R^{2} l_{v}\left[1+\frac{n^{2} k^{2}}{4}\left(1+\frac{\sin \left(2 k l_{v}\right)}{2 k l_{v}}\right)\right],
\end{aligned}
$$

with the vessel height $l_{v}$. Therefore, by comparing the volumes of the straight and the wavy vessels the correction for the penetrated distance is given by

$$
l(t)=l_{v}(t)\left[1+\frac{n^{2} k^{2}}{4}\left(1+\frac{\sin \left(2 k l_{v}\right)}{2 k l_{v}}\right)\right] .
$$


Viscosity increase by diffusion

Various adhesives contain solvents like water, whose concentration $C$ in the mixture changes with time due to their diffusion into the cellular structure through vessel walls. To take this effect into account, the solution of the diffusion equation in cylindrical $z-r-\theta$ coordinates can be written as

$$
C(r, \theta, t)=\frac{N_{0}}{4 \pi D t} \exp \left(-\frac{r^{2}}{4 D t}\right)
$$

with the initial amount of solvent $N_{0}$ and the diffusivity $D$. To apply this equation to the case of the study, one needs to distinguish between two phenomena: the evolution of the concentration inside the mixture, and the diffusion into the cellular structure through vessel walls. For simplicity, the first one is neglected. Therefore, the concentration of the solvent inside the mixture is considered to be always homogeneous, and the mixture to loose only solvent through the vessel walls. The mean value for the solvent concentration inside the vessel is taken as

$$
\bar{C}=\frac{1}{\pi R^{2}} \int_{0}^{2 \pi} \int_{0}^{R} C(r, \theta, t) r d r d \theta=C_{0}\left[1-\exp \left(-\frac{R^{2}}{4 D t}\right)\right] .
$$

Now $D$ is just the diffusivity of the solvent into the cell structure and $C_{0}=N_{0} / \pi R^{2}$ is the mean value of the initial concentration inside the vessel.

To obtain the overall equation for the evolution of the viscosity, the concentration evolution $\bar{C}$ is inserted into the respective concentration dependent parameters in Eq. 1. The diffusion of low molar mass portions of the adhesive is not taken into consideration here. Also, the effect of swelling of the wood skeleton due to moisture changes is neglected, since the size of vessels is rather big compared to the size of the tracheids.

\section{Penetration into the network}

The adhesive penetration is dominated by the flow inside the vessel network, hence its topology determines the adhesive distribution. The network is formed by bundles of vessels that weave around rays of various sizes. Inside the bundle, vessels interconnect by contact zones when touching each other (Hass et al. 2010; Bosshard and Kucera 1973). Disorder in the network can only be considered through a numerical approach. Consequently for an analytical model, average topological network parameters have to be used neglecting possible disorder. For this study, a regular network with the average topological parameters $a$ and $b$ for the connectivity in tangential directions and $c, d$ for the connectivity in radial direction is established. Figure 1 shows the vessel network in three dimensions with the geometrical parameters $a, b, c$, and $d$. The two parameters $a$ and $b$ can be obtained from the size distribution of big and middle-sized rays that are mainly responsible for the splitting and joining of the bundles of vessels. The parameters $c$ and $d$, however are more difficult to obtain. Basically, the probability for radial network 
interconnections depends on the vessel density, which is defined by the number of vessels per unit of area in the $R-T$ plane. It is therefore possible to find a relation between the vessel density and the parameters $c$ and $d$. Unfortunately, only $c$ can be obtained experimentally via the porosity. However, it can be assumed that $c \ll d$, which can be interpreted as a significantly lower probability of interconnection in radial than in tangential direction. To obtain the structure parameter $d$, one has to fit one experimental penetration depth. Note that the parameter $d$ is then constant for all other penetration process into wood of the same species. However, the sensitivity of $d$ on the results proved to be rather small in the case of this study. By separating the two geometric parameter sets $a, b$ and $c, d$, a measure for the anisotropic transport in the three principal directions, $L, R, T$ can be obtained (see Fig. 1). Inclined samples with respect to the principal axis can be considered after rotation.

The model is also based on the assumption that the adhesive can move from one vessel to the other one at the connecting points without restriction, which means that there is no cell wall in between at the connection points. A cell wall would decrease or even hinder a free flow of the adhesive; or only part of the adhesive, mainly with smaller molar mass, like water or solvents or low molar mass species in the adhesive, might migrate from one vessel to the other one. Therefore, for simplicity and to obtain an analytical model, the cell wall is not considered in the present approach.

To describe the penetration process of adhesives into wood, the bond line has to be defined following Kamke and Lee (2007) as the whole region, where the adhesive can be found. This includes (1) the pure adhesive layer between the two adherents, and (2) the area, where the adhesive has penetrated into the wood structure (interphase). The adherents are the two pieces of wood, which have been connected by the adhesive. In the case reported here, the focus will be put on the zone where the adhesive layer and the adherent structure coexist. The procedure to obtain the maximum penetration depth includes the calculation of the penetration separately in each principal direction $(L, R, T)$ followed by the application of a rotation matrix to find the total penetration depth of the adhesive when the growth ring angle and the angle between the vertical axis and the longitudinal axis of specimen differs from zero.

Due to the choice of a regular network, a unit cell consisting of two single vessels with interconnections in the vertices and the center of the cell can be used (see Fig. 1). Consequently, Eq. 7 for single vessels with wave number $k=2 \pi / b$, amplitude $n=a / 4$, and length $l_{v}=b$ can be used to obtain the volume of the unit cell

$$
V_{u}=2 A b g_{T}, \text { and } g_{T}=1+\frac{a^{2} \pi^{2}}{16 b^{2}}
$$

with the area $A=\pi R^{2}$. Because the unit cell can reproduce the whole network, it can be used to simplify the calculation of the penetration depth in each direction and, using geometric properties, the adhesive volume within the network, the network parameters and the penetration depth can be calculated. 

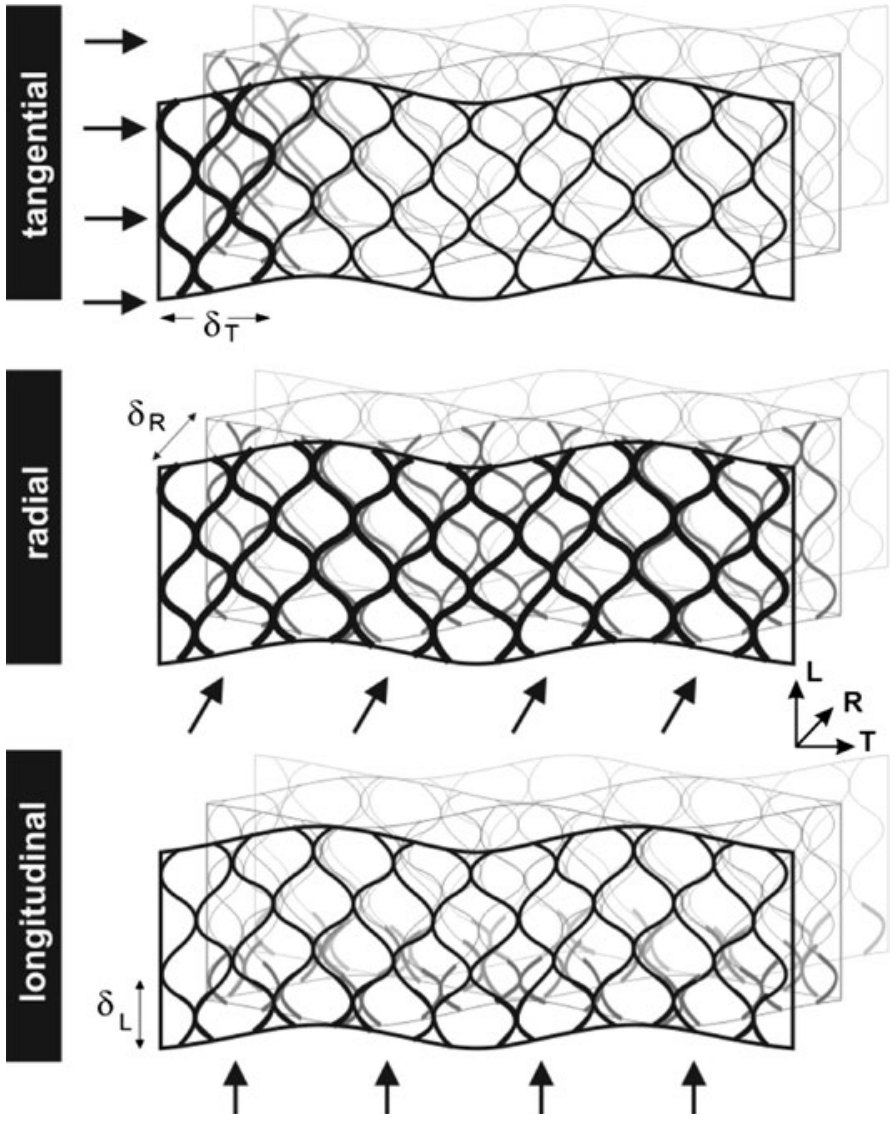

Fig. 2 Adhesive penetration into the vessel network with respective depths in tangential $\left(\delta_{T}\right), \operatorname{radial}\left(\delta_{R}\right)$, and longitudinal $\left(\delta_{L}\right)$ directions. Thick lines represent filled vessels

\section{Penetration in tangential direction}

In order to consider the penetration in tangential direction (see Fig. 2), additionally to the tangential waviness with the wavelength $b$ and the amplitude $a / 4$, the radial waviness with the amplitude $c / 4$ and the wavelength $d$ have to be considered. Figure 2 illustrates, how the vessel network is filled by the adhesive. The path along one radial wave (one layer) $s_{b}\left(x_{T}\right)$ as a function of the tangential coordinate $x_{T}$ can be calculated by integrating

$$
s_{b}=\int_{0}^{x_{T}} \sqrt{1+\frac{\pi^{2} c^{2}}{4 d^{2}} \cos ^{2}\left(\frac{2 \pi x}{d}\right)} d x .
$$

Due to the approximation $c \ll d$, Eq. 12 can be written as 


$$
s_{b} \simeq \int_{0}^{x_{T}}\left[1+\frac{\pi^{2} c^{2}}{8 d^{2}} \cos ^{2}\left(\frac{2 \pi x}{d}\right)\right] \mathrm{d} x
$$

which gives

$$
s_{b}=x_{T}\left[1+\frac{1}{2}\left(g_{R}-1\right)\left(1+\frac{d}{4 \pi x_{T}} \sin \left(\frac{4 \pi x_{T}}{d}\right)\right)\right] \text { with } g_{R}=1+\frac{c^{2} \pi^{2}}{8 d^{2}} .
$$

The number of layers accessible from the bond line is defined by $2 L_{R} / c$ with $L_{R}$ as sample width. Summing $s_{b}$ over all accessible layers gives the total penetrated length $s_{\text {total }}$ as a function of $x_{T}$

$$
s_{\text {total }}=\frac{2 L_{R}}{c} s_{b}\left(x_{T}\right) .
$$

In order to obtain the total penetrated volume $V_{T}$, the number of unit cells $N_{u T}$ along $s_{\text {total }}$ and in longitudinal sample direction $L$ have to be calculated, leading to $N_{\mathrm{uT}}=s_{\text {total }} L_{L} / a b$, and have to be multiplied by the unit cell volume described by Eq. 11, namely

$$
V_{T}\left(x_{T}\right)=N_{\mathrm{uT}} V_{u} .
$$

If the penetration of the adhesive is smaller than the total radial wavelength $d$, $d \sin (4 \pi x / d) / 4 \pi x \approx 1$, and by using Eqs. 11 and 15, $V_{T}$ can be expressed as

$$
V_{T}\left(x_{T}\right)=x_{T} \frac{4 A}{a c} L_{R} L_{L} g_{T} g_{R}
$$

When the adhesive stops to penetrate, this volume becomes the maximum volume $V$ inside the structure and the tangential coordinate $x_{T}$ transforms into the maximum penetration depth $\delta_{T}$,

$$
\delta_{T}=\frac{\mathrm{Vac}}{4 A L_{R} L_{L} g_{T} g_{R}} .
$$

\section{Penetration in radial direction}

Following the idea of calculating the adhesive penetration in each principal direction, the next step is to obtain the penetration depth $\delta_{R}$ when the adhesive penetrates only in radial direction (see Fig. 2). Analogously, the volume occupied by vessels can be counted as a function of the radial coordinate $x_{R}$. Again the total length $s_{\text {total }}$ of the radial wave is calculated, but now as a function of $x_{R}$ by $s_{\text {total }}=2$ $x_{R} / c s_{b}\left(L_{T}\right)$. The number of unit cells can be obtained as

$$
N_{\mathrm{uR}}\left(x_{R}\right)=\frac{2 x_{R}}{c} \frac{s_{b}\left(L_{T}\right)}{a} \frac{L_{L}}{b} .
$$

The total volume occupied is given by multiplying the number of unit cells $N_{\mathrm{uR}}$ (Eq. 19) by the volume $V_{u}$ from Eq. 11, leading to 


$$
V_{T}\left(x_{R}\right)=V_{u} N_{\mathrm{uR}}=x_{R} \frac{4 A}{a c} L_{L} s_{b}\left(L_{T}\right) g_{T} .
$$

As before, $V_{T}\left(x_{R}\right)$ with the volume occupied by the adhesive $V$ must now be compared with the maximum penetration depth in the radial direction $x_{R}=\delta_{R}$ in order to obtain

$$
V=\delta_{R} \frac{4 A}{a c} L_{L} s_{b}\left(L_{T}\right) g_{T}, \text { and } \delta_{R}=\frac{\mathrm{Vac}}{4 A L_{L} s_{b}\left(L_{T}\right) g_{T}} .
$$

Finally, the penetration path $s_{b}$ from Eq. 14 can be inserted to obtain

$$
\delta_{R}=\frac{\text { Vac }}{4 A L_{L} L_{T}\left[1+\frac{1}{2}\left(g_{R}-1\right)\left(1+\frac{d \sin \left(\frac{4 \pi L_{T}}{d}\right)}{4 \pi L_{T}}\right)\right] g_{T}} .
$$

\section{Penetration in longitudinal direction}

Figure 2 shows that the adhesive basically penetrates along the vessels when subject to longitudinal penetration. This value again can be calculated using the number of total unit cells in the plane TR

$$
N_{\mathrm{uL}}=\frac{2 L_{R}}{c} \frac{s_{b}(L x)}{a} .
$$

Multiplying $N_{\mathrm{uL}}$ with the occupied volume $V\left(x_{L}\right)$ of the adhesive for each vessel as a function of $x_{L}$ (Eq. 7), and taking into account that the penetration $x_{L} \ll b, b$ $\sin \left(4 \pi x_{L} / b\right) / 4 \pi x_{L} \approx 1$, one obtains

$$
V_{T}\left(x_{L}\right)=x_{L} \frac{4 A}{a c} L_{R} s_{b}\left(L_{T}\right)\left(2 g_{T}-1\right) .
$$

By comparing this volume with the adhesive volume (Eq. 14), the maximum penetration depth $\delta_{L}$ can again be expressed as

$$
\delta_{L}=\frac{\operatorname{Vac}}{4 A L_{R} L_{T}\left[1+\frac{1}{2}\left(g_{R}-1\right)\left(1+\frac{d \sin \left(\frac{4 \pi L_{T}}{d}\right)}{4 \pi L_{T}}\right)\right]\left(2 g_{T}-1\right)} .
$$

To summarize, the maximum penetration depths in the three principal directions could be obtained (Eqs. 18, 22, and 25). For practical reasons, expressing $\delta_{R, L, T}$ in terms of the porosity is advantageous as it simplifies the model verification. The porosity $\varepsilon$ of the wood can be extracted easily from experimental data (Hass et al. 2010). The number of vessels $N_{v}$ in the $R T$ plane equals $N_{v}=2 N_{\mathrm{uL}}$ (each cell unit has two vessels). The porosity is therefore

$$
\varepsilon=\frac{N_{v} A}{L_{R} L_{T}}=\frac{4 A}{a c}\left[1+\frac{1}{2}\left(g_{R}-1\right)\left(1+\frac{d \sin \left(\frac{4 \pi L_{T}}{d}\right)}{4 \pi L_{T}}\right)\right] .
$$

Considering the length of the sample in the tangential direction $L_{T}$ to be larger than the radial wavelength $d$, the periodic part on the right side of Eq. 26 can be neglected, leading to 


$$
\varepsilon=\frac{2 A}{a c}\left(g_{R}+1\right) .
$$

Inserting $\varepsilon$ into Eqs. 18, 22, and 25, and using the approximation $d \ll L_{T}$, the maximum penetration depths become

$$
\delta_{R}=\frac{V}{\varepsilon L_{L} L_{T} g_{T}}, \delta_{L}=\frac{V}{\varepsilon L_{R} L_{T}\left(2 g_{T}-1\right)}, \delta_{T}=\frac{V\left(g_{R}+1\right)}{2 \varepsilon L_{R} L_{L} g_{T} g_{R}} .
$$

\section{Limitation due to the total amount of applied adhesive}

Up to now, the penetration of an infinite amount of non-hardening fluid was calculated. However, the amount of applied adhesive and the penetration time due to hardening are both limited. Therefore, the volume $V$ needs to be calculated considering these limitations. Both will lead to different penetrated volumes, however the one resulting in a smaller penetration is the decisive one. To calculate the penetrated volume, the $L, R, T$-directions must be treated separately.

For tangential penetration, Eq. 28 is used and only the application of the adhesive on the $R L$ plane is considered. From there, the adhesive can penetrate into two channels with radius $R$ per unit cell. Considering the number of unit cells on this face $N_{u T f}=2 L_{L} L_{R} / c b$, the penetrated volume after the hardening process, using Eq. 4 , is

$$
V_{\mathrm{RL}}=4 V_{p} \frac{L_{L} L_{R}}{c b},
$$

with $V_{p}=\pi R^{2} l(t)$. Inserting Eq. 29 into Eq. 28 and using Eq. 26, the penetration depth with hardening $\delta_{\mathrm{Th}}$ can be obtained as

$$
\delta_{\mathrm{Th}}=\frac{a}{b} \frac{l(t)}{g_{T} g_{R}} .
$$

For the radial penetration, the adhesive can penetrate into four channels with radius $R$ per unit cell; considering the number of unit cells on this face $N_{u R f}=2 L_{T}$ $L_{L} / d b$, the penetrated volume is given by,

$$
V_{\mathrm{LT}}=4 V_{p} \frac{L_{T} L_{L}}{d b} .
$$

Inserting $V_{\mathrm{LT}}$ into Eq. 28 and using the approximation $d \ll L_{T}$ yields

$$
\delta_{\mathrm{Rh}}=\frac{8}{\pi} \frac{a}{b} \frac{\sqrt{\frac{1}{2}\left(g_{R}-1\right)} l(t)}{\left(g_{R}+1\right) g_{T}} .
$$

Finally, for the longitudinal penetration, $\delta_{\mathrm{Lh}}$, following a similar procedure, with $N_{u L f}=2 L_{R} L_{T}\left(g_{R}+1\right) / a c$ as the number of cell units on this face and the penetrated volume $V_{\mathrm{RT}}=2 L_{R} L_{T}\left(g_{R}+1\right) V_{p} / a c$, the penetration depth becomes

$$
\delta_{\mathrm{Lh}}=\frac{l(t)}{\left(2 g_{T}-1\right)} .
$$


These values determine the maximum penetration depth that the adhesive can reach before solidifying. However, it is also possible, that not enough adhesive is available, and penetration stops before. Using the available adhesive volume $V$ in Eqs. 28, the penetration depths $\delta_{R}, \delta_{T}$, and $\delta_{L}$ can be calculated and compared to the hardening ones $\left(\delta_{\mathrm{Rh}}, \delta_{\mathrm{Th}}, \delta_{\mathrm{Lh}}\right)$, e.g., if $\delta_{R}<\delta_{\mathrm{Rh}}$, to obtain the limiting case.

\section{Penetration depth for arbitrary orientations}

In order to apply the model to real situations, the deviation of the adhesive application surface from the wood material system must be considered. Therefore, the global penetration depth $\delta_{V}$ and $\delta_{h}$ must be calculated as a function of $\delta_{R}, \delta_{T}, \delta_{L}$, and of $\delta_{\mathrm{Rh}}, \delta_{\mathrm{Th}}, \delta_{\mathrm{Lh}}$, respectively. This can be made via a rotation matrix with the growth ring angle $\psi$ and the angle $\theta$ between the vertical axis and the longitudinal axis of the specimen. Assuming that the adhesive is always applied on the $Y Z$ plane, two rotations in the principal coordinate system are used: one in the radial direction and the other in the longitudinal direction via the rotation matrix

$$
\underline{\underline{M}}=\left(\begin{array}{lll}
\sin (\psi) & -\cos (\theta) \cos (\psi) & -\cos (\psi) \sin (\theta) \\
\cos (\psi) & \cos (\theta) \sin (\psi) & \sin (\theta) \sin (\psi) \\
0 & -\sin (\theta) & \cos (\theta)
\end{array}\right) .
$$

It has to be noted that Eq. 28 gives a dependence of the penetration depths on the application surfaces RT, LT, LR. To consider arbitrary application surfaces, a penetration vector

$$
\underline{\delta}=\left(\Delta_{R}, \Delta_{T}, \Delta_{L}\right) \equiv \frac{V}{\varepsilon}\left(\frac{1}{g_{T}}, \frac{\frac{1}{2}\left(g_{R}+1\right)}{g_{T} g_{R}}, \frac{1}{2 g_{T}-1}\right),
$$

is defined in the body fixed coordinate system (LRT) and rotated using the rotation matrix $\underline{\underline{M}}$ to the vector $\underline{\delta}$ in global $(X Y Z)$ coordinates. The $X$ component gives the maximum penetration depth $\delta_{V}$

$$
\delta_{V}=\frac{\Delta_{T}}{A_{\mathrm{ad}}} \cos (\theta) \sin (\psi)+\frac{\Delta_{L}}{A_{\mathrm{ad}}} \sin (\theta) \sin (\psi)+\frac{\Delta_{R}}{A_{\mathrm{ad}}} \cos (\psi),
$$

with the area $A_{\text {ad }}$ of the surface where the adhesive is applied. The rotation matrix can be directly applied to the vector $\underline{\delta}_{h}=\left(\delta_{\mathrm{Rh}}, \delta_{\mathrm{Th}}, \delta_{\mathrm{Lh}}\right)$ giving

$$
\delta_{h}=\delta_{\mathrm{Th}} \cos (\theta) \sin (\psi)+\delta_{\mathrm{Lh}} \sin (\theta) \sin (\psi)+\delta_{\mathrm{Rh}} \cos (\psi) .
$$

With these derivations, the geometric and dynamical description of the model is completed. The information about the dynamics of the adhesive is included in the length $l(t)$ according to Eq. 4. Finally, the maximum penetration depth with solvent diffusion can be calculated using Eq. 37, by replacing the concentration function in Eq. 1 for the respective adhesives.

To finalize the description of the model, the main underlying geometrical and physical assumptions and approximations are summarized as follows:

Geometrical approximations mainly address the pore space geometry. The complicated geometry of hardwoods is idealized as a network composed of wavy 
vessels of identical size and shape. Therefore, porosity is considered to be homogeneous over the sample. To simplify the trigonometric expressions arising from the waviness of vessels, $a \ll b$ and $c \ll d$, expressing that the vessel length is significantly larger than the amplitude of oscillation, and that therefore the probability of a vessel interconnection in radial direction is very small compared to the tangential one. For the network, the same approximation is made, namely that the radial wavelength $d$ is much smaller than the tangential sample length $L_{T}$. The physical assumptions made are necessary to formulate an analytically solvable model and mainly address adhesive properties such as the neglect of solvent diffusion inside the adhesive leading to a homogeneous viscosity of the adhesive inside the vessel. Also, the elastic and hygric response of the cellular structure on pressure and diffusive solvents is neglected for simplicity, and it is considered that the adhesive can move from one vessel to the other one at the connecting points without restriction, which means that there is no cell wall in between at the connection points.

In a next step, the model is applied to experiments described by Hass et al. (2011).

\section{Application of the model}

Using synchrotron radiation, X-ray tomographic microscopy (SRXTM) and digital image analysis, bond lines can be segmented from tomographic images of beech samples bonded with PUR, UF, and PVAc adhesives of different viscosity under growth ring angles ranging from $0^{\circ}$ to $90^{\circ}$ in $15^{\circ}$ steps (Hass et al. 2011). Since the model described above is periodic, the maximum penetration depths will be calculated for various situations. Calculation procedure is as follows: First, the penetration distance $l(t)$ of the adhesive inside a single vessel is calculated. Note that for PUR the calculation is without time dependence of the concentration using Eq. 5. Contrary, for PVAc and UF time dependence according to Eq. 10 is inserted in the calculation. The porosity $\varepsilon$ and the mean radius of the vessel $R$ are taken from an earlier SRXTM study (Hass et al. 2010) as $R=28.03 \mu \mathrm{m}$ and porosity $\varepsilon=0.34$. For all samples, the average pressure applied was $P_{A}=0.7 \mathrm{MPa}$ (Hass et al. 2011). According to literature, the surface tension $\sigma$ (Bhattacharya and Ray 2004; Hse 1972; Lee et al. 2007), for the three types of adhesives is not large enough to compete with the applied pressure term in Eq. 3. This leads to negligible capillarity effects with the consequences $\mu=P_{A}$. The parameters for the viscosity $\eta_{g}, \alpha, \beta$, and $\gamma$ are taken from Hass et al. (2011).

- For PUR adhesive, the following parameters were chosen: concentration $C_{0}=0.71 \mathrm{~cm}^{3} / \mathrm{gr} ; \eta_{g}=4,911 \mathrm{mPa} \cdot \mathrm{s} ; \gamma=9.74 \times 10^{-5} ; \alpha=0.0028 \mathrm{~s}^{-1}$, and $\beta=0$. The diffusion of solvent is not relevant. With this set of parameters, the penetration length for PUR $l(t)_{\mathrm{PUR}}$ is calculated using Eq. 5 to $l(t)_{\mathrm{PUR}}=0.304 \mathrm{~m}$. This value is huge compared with the size of the wood samples, however, it relates to the path along the waving vessels that can easily reach lengths of $0.5 \mathrm{~m}$ and above. 

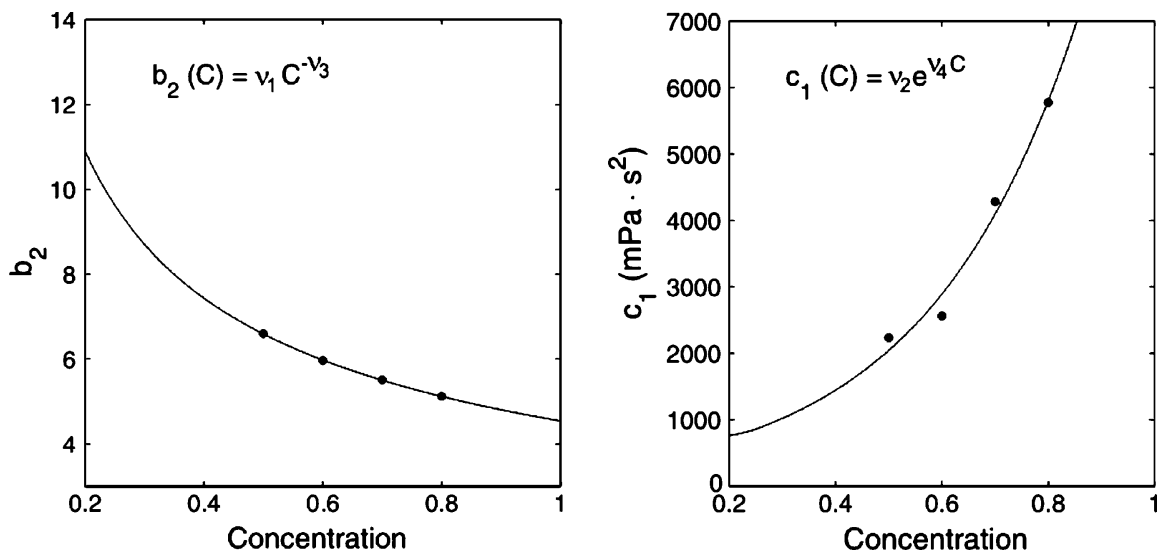

Fig. 3 Dependence of the viscosity parameters $b_{2}$ and $c_{1}$ on the water portion for the UF adhesive used. The dots denote the experimental data and the solid line the exponential fit. Experimental values for UF are $v_{1}=4.542, v_{2}=0.5379, v_{3}=358.3, v_{4}=3.482$

- In the case of PVAc, the parameters are $C_{0}=0.49 ; \eta_{\mathrm{g}}=0.001859 \mathrm{mPa} \mathrm{s}$; $\gamma=0 ; \alpha=0 \mathrm{~s}^{-1}$; and $\beta=29.64$. The diffusivity of the solvent (water) for the samples is taken as $D=3.0 \times 10^{-9} \mathrm{~m}^{2} \mathrm{~s}^{-1}$ diffusivity (Kurjatko and Kudela 1998; Olek et al. 2005). Using Eq. 4, a significantly lower penetration length $l(t)_{\mathrm{PVAC}}=0.7 \mathrm{~mm}$ was found.

- For UF, the viscosity parameters $b_{2}$ and $c_{1}$ of Eq. 2, which change with the solvent concentration, were included in the calculation. The experimental viscosity data from Hass et al. (2011) were fitted with analytical curves (see Fig. 3) in order to determine the concentration dependence of the viscosity parameters $b_{2}$ and $c_{1}$. After the identification of the correct values for $b_{2}$ and $c_{1}$, Eq. 4 was integrated, and a vessel penetration depth of $l(t)_{\mathrm{UF}}=1.1 \mathrm{~mm}$ was obtained.

The vessel geometry parameters $a$ and $b$ were determined experimentally using image processing (Hass et al. 2011). For this work, the area and the eccentricity of segmented rays were measured and averaged over several samples yielding $a=0.156 \mathrm{~mm}$ and $b=1.574 \mathrm{~mm}$. The cylindric samples were $10 \mathrm{~mm}$ high with a diameter of $3 \mathrm{~mm}$, leading to an adhesive area of $A_{a d}=30 \mathrm{~mm}^{2}$. As described in Hass et al. (2011), the quantity of applied adhesive was around $200 \mathrm{~g} / \mathrm{mm}^{2}$ for all adhesives.

As next step, the maximum penetration depths for samples with different growth ring $\psi$ and grain angles $\theta$ (see Figs. 4 and 5) are compared. The parameter $d$ was fitted to obtain $g_{R} \simeq 1$, what can be interpreted as a very low probability of interconnection in radial compared to the tangential direction and by using Eq. 27, a value for the parameter $c=186.2 \mu \mathrm{m}$ was obtained.

- For PUR, a sample with angles, $\psi=50^{\circ}$ and $\theta=3.85^{\circ}$ was chosen. Calculating the maximum penetration depth via Eq. 36 leads to a value of $\delta_{h}=38.5 \mathrm{~mm}$ with hardening as limiting factor. However, using the volume limitation with 

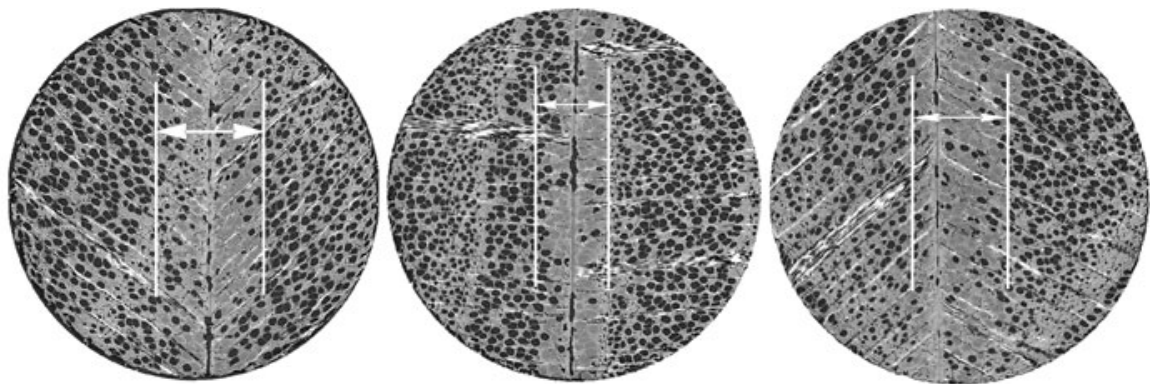

\begin{tabular}{|llll|}
\hline $2 \delta[\mu \mathrm{m}]$ & 848 & 596 & 811 \\
$\psi\left[^{\circ}\right]$ & 50 & 1.1 & 27.9 \\
$\theta\left[^{\circ}\right]$ & 3.85 & 3.6 & 3.8 \\
\hline
\end{tabular}

Fig. 4 PUR bond lines in beech samples. The maximum penetration depth predicted by the model for all samples with growth ring angles $\psi$ and grain angle $\theta$ is shown by the white lines

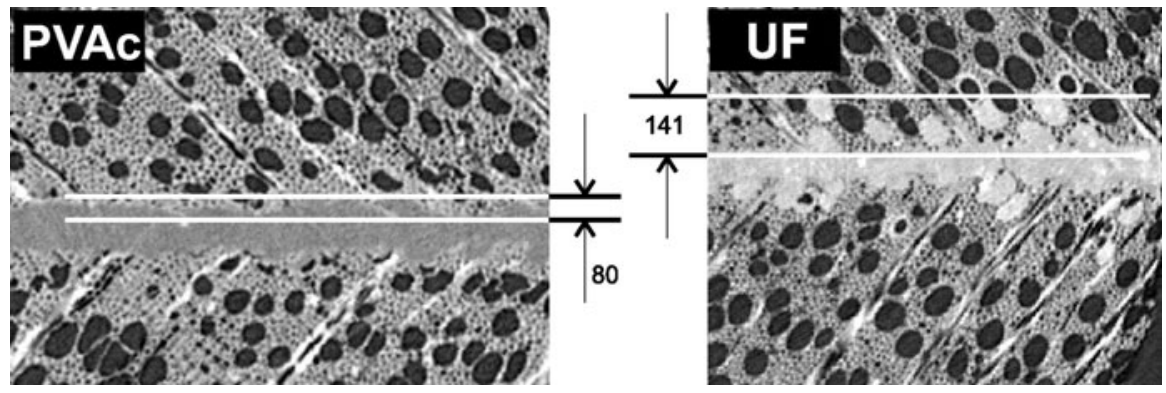

Fig. 5 Bond lines of PVAc and UF adhesive in beech with predicted maximum penetration depth for samples with the orientation angles $\psi$ and $\theta$. All dimensions are given in $\mu \mathrm{m}$

Eq. 37, $\delta_{\mathrm{V}}=848 \mu \mathrm{m}$ is obtained. Therefore, it can be concluded that the entire applied adhesive penetrated before hardening, leading to a starved bond line (see Fig. 4). Note that the adhesive penetrates both wood pieces, but significantly deeper on the application side (right side of samples in Fig. 4). This is a consequence of capillarity effects since adhesion was only applied on one side. It seems that the assumption that capillary pressure is much smaller than the applied pressure does not hold for the utilized PUR pre-polymer. During the adhesive application no pressure was applied, and therefore, the assumption of this study that applied pressure is much larger than capillarity forces, in Eq. 3, is not valid in this initial time interval. Nevertheless, for the case of PUR, where all the adhesive penetrates into the wood structure, the removal of this does not change the final result, and an average value of $\delta=424 \mu \mathrm{m}$ was used showing good agreement with the experimental data. In order to test the model for other orientations $(\psi, \theta)$, a sample with $\psi=1.1^{\circ}$ and $\theta=3.6^{\circ}$ was chosen, hence using the previous calculation but applying a new rotation matrix $\underline{\underline{M}}$. The results were penetration depth $\delta_{V}=596 \mu \mathrm{m}, \delta=298 \mu \mathrm{m}$, and $\delta_{h}=1.32 \mathrm{~mm}$. Figure 4 shows the quality of the analytical prediction. Repetition of the 
calculation for angles $\psi=27.9^{\circ}$ and $\theta=3.8^{\circ}$ gave the penetration depths $\delta_{V}=811 \mu \mathrm{m}, \delta=405.5 \mu \mathrm{m}$, and $\delta_{h}=23.6 \mathrm{~mm}$ (compare with Fig. 4). These tests show that the developed model is a good approximation for the beech structure; under this condition the network parameters $a, b, c$, and $d$ could be fixed for further calculations.

- The developed model was also checked concerning the penetration of PVAc using a sample oriented at angles $\psi=46.1^{\circ}$ and $\theta=3.4^{\circ}$ and calculating the maximum penetration depths based on Eqs. 36 and 37 . The result was $\delta_{V}=851 \mu \mathrm{m}$, leading to $\delta=425.5 \mu \mathrm{m}$ and $\delta_{h}=80 \mu \mathrm{m}$. In this case, the maximum penetration depth is limited by the solidification process. Figure 5 shows that almost all the adhesive remains in the bond line with only a small quantity of adhesive inside the vessel network.

- For UF, the same procedure as before was repeated on a sample with orientation angles $\psi=37.2^{\circ}$ and $\theta=4.3^{\circ}$, yielding penetration depths $\delta_{V}=856 \mu \mathrm{m}$, $\delta=428 \mu \mathrm{m}$, and $\delta_{h}=141 \mu \mathrm{m}$. Again the penetration of the adhesive is limited by adhesive hardening. Figure 5 shows the sample with the predicted penetration depth, exhibiting excellent agreements between the analytical prediction and the experimental results.

In order to investigate the dependence of the maximum penetration depth from the growth ring angle $\psi, \psi$ was varied whereas all other parameters were held constant as in the example described above for UF. Figure 6 shows the two limiting conditions for the penetration depth. In the case that hardening limits penetration, the penetration depth increases with the growth ring angle; for the case that the maximum available volume is the limitation in the penetration, a distinct maximum at approximately $48^{\circ}$ is given. This observation is in agreement with results for PUR, where also the applied amount of adhesive was the limitation, as proven by Hass et al. (2011). This result shows that even though the wood anatomy was simplified to a homogeneous and regular network for the adhesive transport, beech seems to be well described by the model. For a desired penetration depth, the model can predict the optimal growth ring angle of the samples.

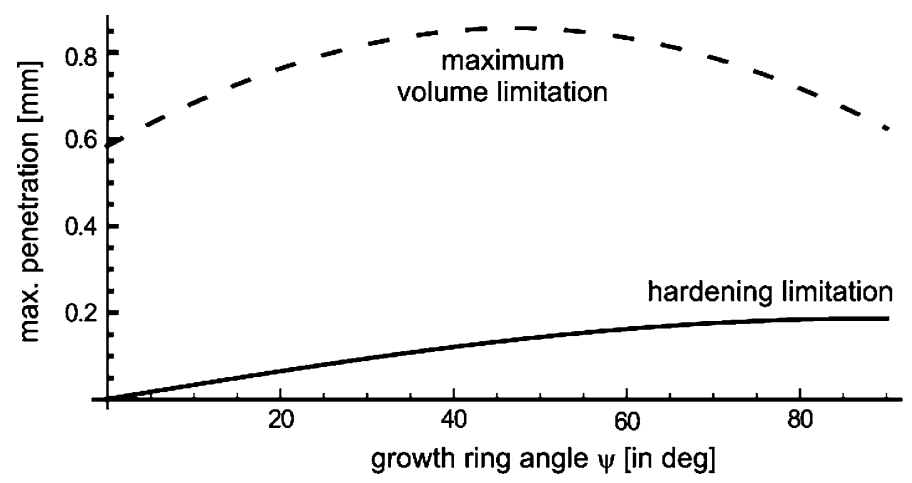

Fig. 6 Dependence of the penetration depth on the growth ring angle for the two cases of limitation of the penetration (1) by the hardening reaction (solid line) and (2) by the limited amount of adhesive applied (dotted line) 

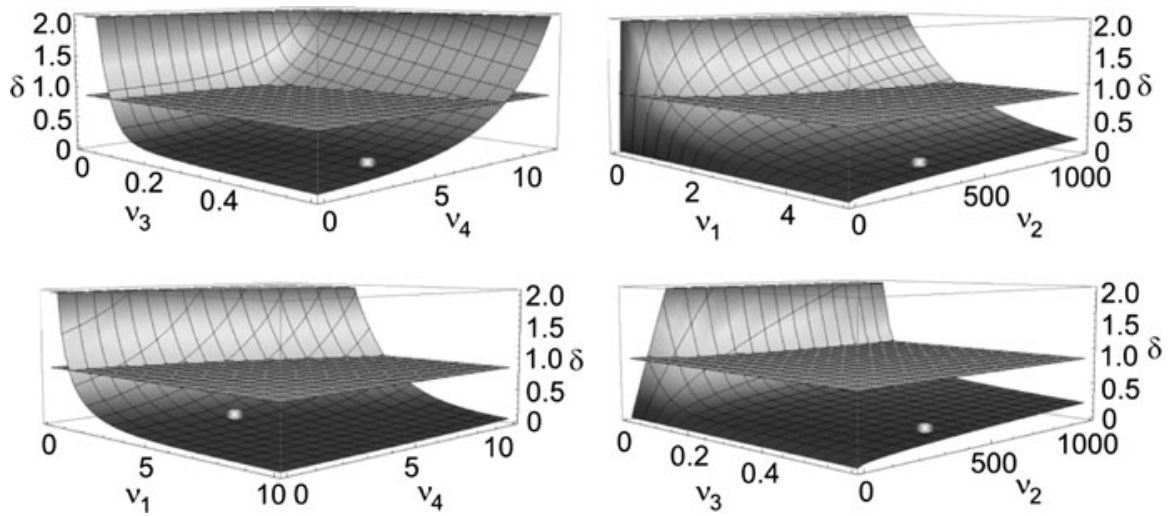

Fig. 7 Penetration depth $\delta(\mathrm{mm})$ as a function of adhesive parameters from Fig. 3 for UF and a growth ring angle of $45^{\circ}$. The experimentally obtained value for UF is marked by the white dot. (color version online)

The model can also be used in the design of new adhesives with optimized properties, like reactivity, if an ideal penetration depth is to be reached. Figure 7 shows the maximum penetration depth for a wide range of the adhesive parameters $v_{1 \ldots 4}$ from Fig. 3. The horizontal planes in Fig. 7 represent the case where all available adhesive has penetrated into the vessel structure, while the curved surfaces give the penetration limit due to adhesive hardening. The intersection curve of the two surfaces separates regions with complete penetration from those, where penetration is limited by adhesive hardening. Therefore, Fig. 7 allows to choose a pair of reactivity parameters in order to obtain a desired penetration depth. The model can also be used to minimize solvent concentration and amount of applied adhesive for a required penetration depth. Figure 8 illustrates the maximum penetration depth as a function of the solvent concentration and the total amount of applied adhesive. The solid lines represent the proportions between solvent concentration and the total applied volume of adhesive which give the same penetration depth. Additional information can also be obtained from the model, when investigating the dependence of the maximum penetration depth on the porosity giving a decreasing curve when the volume is the limiting factor for the penetration (Fig. 9 left). This is expected since for smaller porosity, the vessels are more distant or thinner. For the same amount of adhesive, the penetration distance therefore increases. However, when the hardening process is the limiting factor, the penetration depth increases until it reaches a maximum and then it goes back to zero again. With this last result, the model can be used to predict the penetration for varying porosity, e.g., over the growth ring.

\section{Discussions and conclusion}

An analytical model for the prediction of the penetration depth of adhesives, paints, or hardening fluids in general into the porous structure of beech was presented. The 


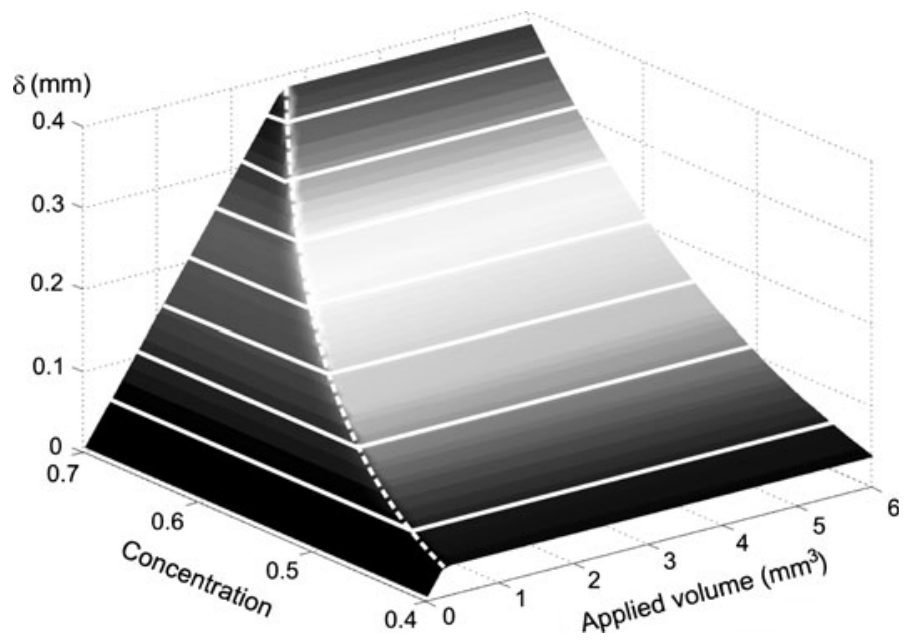

Fig. 8 Penetration depth $\delta(\mathrm{mm})$ as a function of the solvent proportion and total amount of applied adhesive for UF and a growth ring angle of $45^{\circ}$. The solid lines represent a certain penetration depth $\delta$; the dashed line divides the surface into two regions, right for the case that the penetration is limited by the hardening process, and left for the case where the total volume of applied adhesive is the limiting factor. (color version online)

(a)

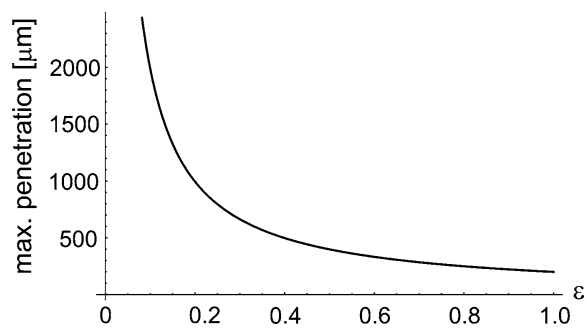

(b)

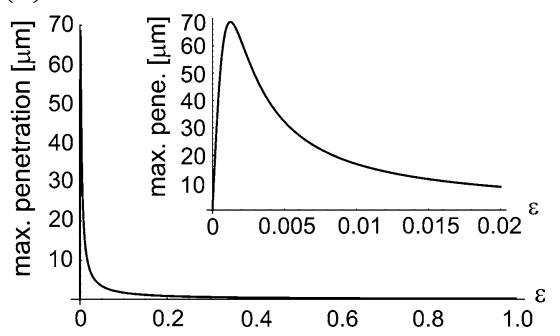

Fig. 9 Penetration depth $\delta(\mu \mathrm{m})$ as a function of the porosity $\varepsilon$ for UF when the total applied volume (a) and the hardening process (b) are the limiting factors

model is focused on hardwood, where the pore space is formed by a network of interconnected vessels. The network is characterized by parameters that are related to the amplitude and the wavelength of the oscillating vessels in tangential and radial directions and to the porosity of wood originating from the vessel network. The results from the model calculations were compared to experimental results, and good agreement for various adhesives was found. The simplified adhesive transport model can therefore describe well the much more disordered, complex pore space of beech.

The analytical model considers generic types of adhesive with different hardening mechanisms. It can be adapted to other fluids by alternative expression for the viscosity dependence on concentration and time. In the work presented here, the model was applied to three major types of adhesives (PUR, UF, and PVAc), and 
the respective penetration depths were compared. For adhesives, whose solidification process depends on the change of concentration, a description for solvent diffusion inside the beech wood was included. This approach enables the application of the model for different adhesives like UF and PVAc. Penetration can be limited by two aspects: (1) the hardening process, stopping the penetration and (2) the total amount of applied adhesive that was available to penetrate into the vessel network. By comparing the model with the experimental data it was shown that it is possible to model the maximum penetration depth for the three different adhesives (PUR, PVAC, and UF).

The model can be used in a broad range of applications. By determining the morphological and rheological parameters, it can be applied to a wide range of wood species and to fluids with various hardening kinetics in order to predict the penetration depth of these fluids into porous structures with capillarity-dominated transport conditions. However, in the future, the model will be applied to different kinds of hardwood species, i.e., ring-porous hardwoods, hardwood that contains some tyloses in the vessels, in order to make it more realistic and suited to make quantitative predictions. Additionally, the level of complexity of the adhesive can be increased as well, and the release of gases during the curing process phenomenon can be included.

Acknowledgments The authors are grateful for the financial support of the Swiss National Science Foundation (SNF) under Grant No. 116052, and SNF SINERGIA under Grant No. CRSI22_125184.

\section{References}

Bhattacharya A, Ray P (2004) Studies on surface tension of poly(Vinyl Alcohol): effect of concentration, temperature, and addition of chaotropic agents. J Appl Poly Sci 93:122-130

Bosshard HH, Kucera L (1973) The network of vessel system in Fagus sylvatica L. Holz Roh- Werkst 31:437-445

Collett BM (1972) A review of surface and interfacial adhesion in wood science and related fields. Wood Sci Technol 6:1-42

Custodio J, Broughton J, Cruz H (2009) A review of factors influencing the durability of structural bonded timber joints. Int J Adhesion Adhesives 29:173-185

Hass P, Wittel FK, McDonald SA, Marone F, Stampanoni M, Herrmann HJ, Niemz P (2010) Pore space analysis of beech wood-the vessel network. Holzforschung 64:639-644

Hass P, Mendoza M, Wittel FK, Niemz P, Herrmann HJ (2011) Adhesive penetration in beech wood: experiments, Wood Sci Technol, pp 1-14. doi:10.1007/s00226-011-0410-6

Hse CY (1972) Surface tension of phenol-formaldehyde wood adhesives. Holzforschung 26:82-85

Kamke FA, Lee JN (2007) Adhesive penetration of wood-a review. Wood Fiber Sci 39(2):205-220

Kurjatko S, Kudela J (1998) Wood structure and properties. Arbora Publisher, Zvolen

Lee S, Shupe TF, Groom LH, Hse CY (2007) Wetting behaviors of phenol- and urea-formaldehyde resins as compatibilizers. Wood Fiber Sci 39:482-492

Marra AA (1992) Technology of wood bonding. Van Nostrand Reinhold, New York

Niemz P, Mannes D, Lehmann E, Vontobel P, Haase S (2004) Untersuchungen zur Verteilung des Klebstoffes im Bereich der Leimfugen mittels Neutronenradiographie und Mikroskopie. Holz RohWerkst 62:424-432

Olek W, Perré P, Weres J (2005) Inverse analysis of the transient bound water diffusion in wood. Holzforschung 59:38-45

Sernek M, Resnik J, Kamke FA (1999) Penetration of liquid urea-formaldehyde adhesive into beech wood. Wood Fiber Sci 31(1):41-48 
Siau JF (1984) Transport processes in wood. Springer, New York

Suchsland O (1958) Über das Eindringen des Leims bei der Holzverleimung und die Bedeutung der Eindringtiefe für die Fugenfestigkeit. Holz Roh- Werkst 16(39):101-108

Wang WQ, Yan N (2005) Characterizing liquid resin penetration in wood using a mercury intrusion porosimeter. Wood Fiber Sci 37:505-514

Washburn EW (1921) The dynamics of capillarity flow. Phys Rev 17:273-283 